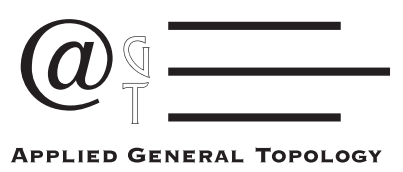

\title{
The Alexandroff Duplicate and its subspaces
}

\author{
Agata Caserta* and Stephen Watson
}

\begin{abstract}
We study some topological properties of the class of the Alexandroff duplicates and their subspaces. We give a characterization of metrizability and Lindelöf properties of subspaces of the Alexandroff duplicate. This characterization clarifies the potential for finding Michael spaces among the subspaces of Alexandroff duplicates.
\end{abstract}

2000 AMS Classification: 54B05, 54B10.

Keywords: Resolution, Alexandroff duplicate, Lindelöf property, Michaeltype line.

In their famous 1922 memoir on compact spaces [1], Alexandroff and Urysohn defined a topological space that has become known as the Alexandroff double circle or the Alexandroff duplicate. In this paper we study several version of the Alexandroff duplicate by viewing it as particular resolution by constant maps.

Alexandroff duplicates have been studied and used by many topologists. In particular, Michael's 1963 example of a Michael spaces is subspace of an Alexandroff duplicate.

This paper is an organized study of the topological properties of the class of the Alexandroff duplicates and of their subspaces. In particular, we characterize when subspaces of Alexandroff duplicates have the Lindelöf property. This suggests that the potential for finding Michael spaces among the subspaces of Alexandroff duplicates is not high.

In this note, $\mathbb{P}$ and $\mathbb{Q}$ denote the set of the irrational and rational numbers, respectively. Ordinal numbers are denoted by Greek letters; when viewed as topological spaces, they are given the order topology. Products of topological spaces are endowed with the standard product topology.

The symbol $[A]^{\lambda}$ denotes the family of subsets of $A$ having size exactly $\lambda$. The symbols $[A]^{\leq \lambda}$ and $[A]^{<\lambda}$ have similar meaning.

${ }^{*}$ Corresponding author. 
Let $\leq_{*}$ be the quasi-order on a countable product of ordered sets that is associated to the coordinate-wise order on each set. Thus $f \leq_{*} g$ stands for $f(n) \leq g(n)$ for all but finitely many $n \in \omega$. A subset of $\omega_{\omega}$ is unbounded if it is unbounded in $\left({ }^{\omega} \omega, \leq_{*}\right)$. A dominating family is an unbounded set that is cofinal in $\left({ }^{\omega} \omega, \leq_{*}\right)$. A subset of ${ }^{\omega} \omega$ is said to be a scale if it is a dominating family and is well-ordered by $\leq_{*}$.

Recall that $\mathbb{P}$ can be identified with ${ }^{\omega} \omega$ with the product topology. For each $\xi \in{ }^{<\omega} \omega=\{\eta \mid \eta:[0, n] \rightarrow \omega$ for some $n\}$, a basic open neighborhood of $\xi$ in the product topology is $\left\{f \in \omega^{\omega} \omega: \xi \subseteq f\right\}$. For every $g \in \omega^{\omega} \omega$, the sets $\left\{f \in \omega_{\omega}: f \leq g\right\}$ and $\left\{f \in \omega_{\omega}: f \leq_{*} g\right\}$ are respectively compact and $\sigma$-compact (see $[14]$ ).

Let $X$ and $Y$ be topological spaces. A set $A \subseteq X$ is $Y$-analytic if it is a projection on $X$ of a closed subset of $X \times Y$. In particular, $A \subseteq X$ is analytic if it is $\mathbb{P}$-analytic.

Given a function $f: X \rightarrow Y$, the small image of $A \subseteq X$ is defined by $f^{\sharp}(A)=\left\{y \in Y: f^{-1}(y) \subseteq A\right\}$. Sometimes we abuse of terminology and say that $f^{\sharp}$ is open, with the meaning that for each open subset $A$ of $X, f^{\sharp}(A)$ is an open subset of $Y$.

In most cases we will employ the notation used in [6] and [9].

\section{BASIC DEFINITIONS AND PRELIMINARY RESULTS}

We begin with the definition of the Alexandroff double circle as a resolution by constant map.

Definition 1.1. Let $(X, \tau)$ be a topological space. For any $U \in \tau$, denote $\hat{U}=U \times 2$. Define a base for a topology on $Y=X \times 2$ by $\mathcal{B}=\mathcal{B}_{0} \cup \mathcal{B}_{1}$, where $\mathcal{B}_{0}$ is the family of all subsets $\hat{U} \backslash(F \times\{1\})$ of $Y$, with $U \in \tau$, and $F \in[X]^{<\omega}$, and $\mathcal{B}_{1}=\{(x, 1): x \in X\}$. This topological space is the resolution of $X$ at each point into the two point space by the constant zero function (see [15] and [7]). However we use the notation $Y=X \times_{a d} 2$ (the subscript ad stands for Alexandroff duplicate).

For each $x \in X$, we denote $\tau(x)=\{U \in \tau: x \in U\}$ and $\mathcal{B}(x)=\{(x, 1)\} \cup$ $\{\hat{U} \backslash\{(x, 1)\}: U \in \tau(x)\}$. Further, let $\mathcal{B}^{\prime}=\bigcup_{x \in X} \mathcal{B}(x)$.

Lemma 1.2. If $X$ is a $T_{1}$ space, then $\mathcal{B}^{\prime}$ is a base, such that $\mathcal{B}^{\prime} \subset \mathcal{B}$, and $\mathcal{B}(x)$ is a local base at each $x \in X$. Moreover, if $Z=(A \times\{1\}) \cup(B \times\{0\}) \subseteq X \times{ }_{a d} 2$ and $\mathcal{U}$ is a base in $X$ at $x \in B \backslash A$, then $\{\hat{U} \cap Z: U \in \mathcal{U}\}$ is a base at $(x, 0)$ in $Z$.

Proof. Let $(x, 0) \in \hat{U} \backslash(F \times\{1\})$. Set $F^{\prime}=F \backslash\{x\}$ and $V=U \backslash F^{\prime}$. Then $\hat{V} \backslash\{(x, 1)\} \subset \hat{U} \backslash(F \times\{1\})$ and $\hat{V} \backslash\{(x, 1)\} \in \mathcal{B}(x)$.

In this paper, unless otherwise stated, topological spaces are considered $T_{1}$. Furthermore, if $\mathcal{U}=\left\{U_{i}: i \in I\right\}$, let $\hat{\mathcal{U}}=\left\{\hat{U}_{i} \mid U_{i} \in \mathcal{U}\right\}$ and $\mathcal{U}^{*}=$ $\left\{\hat{U}_{i} \backslash(F \times\{1\}): F \in[X]^{<\omega}, U_{i} \in \mathcal{U}\right\}$. 
Remark 1.3. The space $X$ is homeomorphic to the subset $Z=(I \times\{1\}) \cup((X \backslash$ $I) \times\{0\})$ of its duplicate $X \times{ }_{a d} 2$. Indeed, the function $\phi: X \rightarrow Z$ defined by $\phi(x)=\left(i d_{X}(x), \chi_{I}(x)\right)$ is clearly a bijection. Now, let $U^{*}=\left(\hat{U} \backslash\left(F_{U} \times\{1\}\right)\right) \cap Z$ be a basic open set in $Z$. Then $\phi^{-1}\left(U^{*}\right)=U \backslash\left(F_{U} \cap I\right)$ which is open in $X$. Moreover, if $U \in \tau$, then $\phi(U)=(U \cap I) \times\{1\} \cup(U \cap(X \backslash I)) \times\{0\}$ is an open set in $Z$.

Given the topological space $X \times_{a d}$, we consider the following functions:

- $r: X \times \times_{a d} 2 \rightarrow X \times\{0\}$ such that for each $x \in X, r(x, 1)=(x, 0)$, and $r\left\{X \times\{0\}=\operatorname{id}_{X \times\{0\}}\right.$;

- $\pi_{0}: X \times_{a d} 2 \rightarrow X$ such that $\pi_{0}(x, i)=x$ for each $x \in X$ and $i=0,1$;

- $\iota: X \rightarrow X \times\{0\} \subset X \times_{a d} 2$ such that for each $x \in X, \iota(x)=(x, 0)$; and its inverse map $\pi_{0}\lceil(X \times\{0\})=\hat{\iota}$.

Note that the projection map $\pi_{0}$ is continuous.

In the following we show some properties of this functions.

Lemma 1.4. Let $X$ be a topological space and $X \times\{0\} \subset X \times \times_{a d} 2$, then $X \cong X \times\{0\}$. Further, $\iota$ and $\hat{\iota}$ are homeomorphisms.

Proof. Let $(\hat{U} \backslash(F \times\{1\})) \cap(X \times\{0\})=U \times\{0\}$ be an open set in the subspace $X \times\{0\}$. Hence $\iota^{-1}(\hat{U} \backslash(F \times\{1\}))=U$, therefore $\iota$ is continuous. The continuity of $\hat{\iota}$ follows from the continuity of $\pi_{0}$. Since $\hat{\iota} \circ \iota=\operatorname{id}_{X}$ and $\iota \circ \hat{\iota}$ $=\operatorname{id}_{X \times\{0\}}$, both $\iota$ and $\hat{\iota}$ are homeomorphisms, i.e., $\iota^{-1}=\hat{\iota}$.

Definition 1.5. Let $Y \subset X$ and $f: X \rightarrow Y$ be a function. Then $f$ is called a retraction of $X$ onto $Y$ ( $Y$ is the retract of $X$ ) if it is continuous and $f \uparrow Y=i d_{Y}$. If $f$ is continuous only at the points of $Y$, then it is called a weak retraction of $X$ onto $Y$.

Lemma 1.6. The map $r$ is a retraction of $X \times_{a d} 2$ onto $X \times\{0\}$. Moreover $r$ is a closed map.

Proof. From $r=\hat{i} \circ \pi_{0}$, it follows that $r$ is a continuous map. Since $r\lceil X \times\{0\}$ $=\operatorname{id}_{X} \times\{0\}, r$ is a retraction of $X \times{ }_{a d} 2$ onto $X \times\{0\}$. We show that $r^{\#}$ is open on $\mathcal{B}$. Let $\hat{U} \backslash(F \times\{1\}) \in \mathcal{B}$. Then $r^{\#}(\hat{U} \backslash(F \times\{1\}))=U \times\{0\} \backslash F \times\{0\}$, which is an open set in $X \times\{0\}$. Thus $r$ is a closed map.

Lemma 1.7. Let $Z=(A \times\{1\}) \cup(B \times\{0\}) \subseteq X \times \times_{a d} 2$ and suppose $\phi: Z \rightarrow$ $B \times\{0\}$ is a continuous map. Define $\phi^{\prime}: Z \rightarrow B \times\{0\}$ by

$$
\phi^{\prime}(x, i)= \begin{cases}\phi(x, 0) & \text { if } x \in A \cap B \wedge i=1 \\ \phi(x, i) & \text { otherwise. }\end{cases}
$$

Then $\phi^{\prime}$ is continuous too. Moreover if $\phi$ is a retraction, then $\phi^{\prime}$ is a retraction too.

Proof. Let $H=Z \backslash((A \cap B) \times\{1\})$ and $K=((A \cap B) \times\{1\}) \cup(B \times\{0\})$ be two closed sets of $Z$. Let $\psi=\phi\lceil H$ and $\theta=r\lceil K$ two continuous maps, such that $\psi\left\lceil K \cap H=\theta\left\lceil K \cap H\right.\right.$. Then, by the pasting lemma, $\phi^{\prime}=\psi \cup \theta$ is a continuous map. Since $\phi\left\lceil B \times\{0\}=i d_{B \times\{0\}}\right.$, then $\phi^{\prime}\left\lceil B \times\{0\}=i d_{B \times\{0\}}\right.$. 
Definition 1.8. Let $X$ and $Y$ be two topological spaces, and $f: X \rightarrow Y$ be a continuous map. Then $f$ is hereditarily closed if $f \nmid Z: Z \rightarrow f(Z)$ is closed, for each subset $Z$ of $X$. The function $f$ is hereditarily perfect if it is hereditarily closed and all fibers $(f \nmid Z)^{-1}(y)$ are compact subsets of $Z$ respectively, for each subset $Z$ of $X$.

Remark 1.9. Observe that for an Hausdorff space $X$, the function $f: X \rightarrow Y$ is hereditarily perfect if and only if $f$ is hereditarily closed and all fibers are finite. Indeed, assume that $\left|(f \mid Z)^{-1}(y)\right| \geq \aleph_{0}$. Let $\left\{z_{n}\right\}_{n}$ be an infinite subset of $\left(f\lceil Z)^{-1}(y)\right.$. Then $\left\{z_{n}\right\}_{n}$ has a cluster point $z$ which is an element of $\left(f\lceil Z)^{-1}(y)\right.$, because it is a closed subset of $Z$. Hence, $\left\{f\left(z_{n}\right)\right\}_{n}$ does not have accumulation point in $f(z)$ which is a contradiction.

Note that for each $Z \subseteq X \times_{a d} 2$ the function $r \nmid Z$ has finite fibers. The following example shows that in general the retraction is not a hereditarily closed map, hence not hereditarily perfect.

Example 1.10. Let $r:[0,1] \times{ }_{a d} 2 \rightarrow[0,1] \times\{0\}$ be a retraction. Let $Z=$ $[0,1) \times 2 \cup\{(1,1)\} \subseteq[0,1] \times a d$, and $F=[0,1) \times 2$. Then $F$ is a closed subset of $Z$ such that $r\lceil Z(F)=[0,1) \times\{0\}$, which is not closed in $r(Z)=[0,1] \times\{0\}$.

Next we show a characterization for a retraction of a subspace of the Alexandroff duplicate. Before we need the following definitions.

Definition 1.11. Let $A$ and $B$ be subsets of a topological space $X$. We say that $A$ is closed in $B$ if for each $b \in B$ such that $b \in \bar{A}$, then $b \in A$.

Definition 1.12. Let $\left\{A_{\alpha}\right\}_{\alpha \in \kappa}, B$ be subsets of a topological space $X$. The family $\left\{A_{\alpha}\right\}_{\alpha \in \kappa}$ is locally finite in $B$ if for each point $b$ of $B$ there exists a neighborhood of $b$ in $X$ which intersects finitely many elements of $\left\{A_{\alpha}\right\}_{\alpha \in \kappa}$.

Lemma 1.13. Let $X$ and $Y$ be topological spaces, $B \subset X$ and $\left\{A_{\alpha}\right\}_{\alpha \in \kappa} a$ family of sets closed in $B$ which is locally finite in $B$. Let $f: B \rightarrow Y$ and for each $\alpha \in \kappa, g_{\alpha}: A_{\alpha} \rightarrow Y$ are continuous maps which are compatible with $f$ and each other. Then $h=f \cup\left(\bigcup_{\alpha \in \kappa} g_{\alpha}\right)$ is a continuous map at each point of $B$.

Proof. Since $h$ is the extension of each $g_{\alpha}$ and $f$, it remains to check the continuity of $h$ at points of $B$. Let $\left(x_{\sigma}\right)_{\sigma \in \Sigma}$ a net, with $b \in B$ such that $x_{\sigma} \rightarrow b$. Then for each neighborhood of $b, I_{X}(b)$, there exists $\sigma_{0} \in \Sigma$ such that for each $\sigma \geq \sigma_{0}, x_{\sigma} \in I_{X}(b)$. By hypothesis the family $\left\{A_{\alpha}\right\}_{\alpha \in \kappa}$ is locally finite in $B$, hence for each $\sigma \geq \sigma_{0}, I_{X}(b) \cap A_{\alpha_{i}}=\varnothing$ for $i \notin\{1, \ldots, n\}$. Set $\Sigma^{\prime}=\left\{\sigma \in \Sigma: \sigma \geq \sigma_{0}\right\}$. Then $\left(x_{\sigma}\right)_{\sigma \in \Sigma^{\prime}} \subseteq B \cup\left(\bigcup_{i=1}^{n} A_{\alpha_{i}}\right)$ and $x_{\sigma} \rightarrow b$. Since every net has a subnet which is an ultranet, we have that if $\mathcal{A}$ is a finite cover of $X$, there exists $A \in \mathcal{A}$ and subnet $\left(x_{\sigma_{\lambda}}\right)_{\lambda \in \Lambda}$ such that $\left(x_{\sigma_{\lambda}}\right)_{\lambda \in \Lambda} \subset A$. Then assume, without loss of generality, that $\left(x_{\sigma_{\lambda}}\right)_{\lambda \in \Lambda} \in B$ or $\left(x_{\sigma_{\lambda}}\right)_{\lambda \in \Lambda} \in A_{\alpha_{i}}$ for some $i \in\{1, \ldots, n\}$. First assume that $\left(x_{\sigma_{\lambda}}\right)_{\lambda \in \Lambda} \in B$. Since $x_{\sigma_{\lambda}} \rightarrow b$ and $\left(h\left(x_{\sigma_{\lambda}}\right)\right)_{\lambda \in \Lambda}=\left(f\left(x_{\sigma_{\lambda}}\right)\right)_{\lambda \in \Lambda}$, by continuity of $f$, follow that $h\left(x_{\sigma_{\lambda}}\right) \rightarrow h(b)$. Let $\left(x_{\sigma_{\lambda}}\right)_{\lambda \in \Lambda} \in A_{\alpha_{i}}$ for some $i \in\{1, \ldots, n\}$. From $A_{\alpha_{i}}$ closed in $B$ and $b \in \overline{A_{\alpha_{i}}} \cap B$, 
it follows that $b \in A_{\alpha_{i}}$. By continuity of $g_{\alpha_{i}}$ we have $g_{\alpha_{i}}\left(x_{\sigma_{\lambda}}\right) \rightarrow g_{\alpha_{i}}(x)$. Thus $h\left(x_{\sigma_{\lambda}}\right) \rightarrow h(b)$.

Note that in the previous Lemma, if the family of $A_{\alpha}$ 's is finite, we only need that all sets $A_{\alpha}$ are closed in $B$.

Definition 1.14. Let $A, B$ be subsets of a topological space $X$ with $A \subseteq B$. We say that $A$ is discrete in $B$ if for each $b \in B$ there exists a neighborhood of $b$ in $X$ which intersects $A$ at most in one point.

Lemma 1.15. Let $X, Y$ be topological spaces and $B \subset X$ such that the points of $\overline{X \backslash B} \cap B$ can be separated by a disjoint family of open sets in $X$. Then there exists $\left\{A_{\alpha}: \alpha \in \kappa\right\}$, with $\kappa=|\overline{X \backslash B} \cap B|$, a family of sets closed in $B$ such that:

(i) $X=\left(\bigcup_{\alpha \in \kappa} A_{\alpha}\right) \cup B$,

(ii) $\left|A_{\alpha} \cap B\right|=1$ for each $\alpha \in \kappa$,

(iii) $A_{\alpha} \cap A_{\beta}=\varnothing$ for each $\alpha$ and $\beta$ distinct.

Moreover, under the additional assumption that $\overline{X \backslash B} \cap B$ is discrete in $B$, it follows that the family $\left\{A_{\alpha}: \alpha \in \kappa\right\}$ is locally finite in $B$.

Proof. Let $\left\{x_{\alpha}: \alpha \in \kappa\right\}=\overline{X \backslash B} \cap B$ and let $\left\{U_{\alpha}: \alpha \in \kappa\right\}$ be a disjoint family of open sets, such that $x_{\alpha} \in U_{\alpha}$ for each $\alpha \in \kappa$. Let $\left\{A_{\alpha}: \alpha \in \kappa\right\}$ be defined by: $A_{\alpha}=\left(U_{\alpha} \backslash B\right) \cup\left\{x_{\alpha}\right\}$ for each $\alpha \in \kappa$ and $A_{0}=\left(X \backslash\left(\bigcup_{\alpha \in \kappa} U_{\alpha} \cup B\right)\right) \cup\left\{x_{\kappa}\right\}$.

Each $A_{\alpha}$ with $\alpha \in \kappa$ is closed in $B$, because the only accumulation points of $X \backslash B$ in $B$ are $x_{\alpha}$ 's and $\left\{x_{\alpha}\right\}=A_{\alpha} \cap B$. Moreover, since $x_{\alpha}$ 's are distinct and $U_{\alpha}$ 's are disjoint, $\left\{A_{\alpha}: \alpha \in \kappa\right\}$ is disjoint.

Assume that $\left\{x_{\alpha}: \alpha \in \kappa\right\}$ is discrete in $B$, we prove that $\left\{A_{\alpha}: \alpha \in \kappa\right\}$ is locally finite in $B$. Note that, for all $\alpha \in \kappa, A_{\alpha} \cap\left(B \backslash\left\{x_{\alpha}: \alpha \in \kappa\right\}\right)=\varnothing$. If $b \in B \backslash\left\{x_{\alpha}: \alpha \in \kappa\right\}$, then $b \notin \overline{X \backslash B}$. Thus there exists a neighborhood $I_{X}(b)$ of $b$ such that $I_{X}(b) \cap(X \backslash B)=\varnothing$. Since $\overline{X \backslash B} \cap B$ is discrete in $B$, there exists a neighborhood $I_{X}^{\prime}(b)$ of $b$ such that $\left|I_{X}^{\prime}(b) \cap\left\{x_{\alpha}: \alpha \in \kappa\right\}\right| \leq 1$. Let $I_{X}^{\prime}(b) \cap\left\{x_{\alpha}: \alpha \in \kappa\right\}=x_{\alpha}$ with $\alpha \in \kappa$. Assume that $\left(I_{X}(b) \cap I_{X}^{\prime}(b)\right) \cap A_{\beta} \neq \varnothing$ for $\beta \in \kappa$ and $\beta \neq \alpha$. Let $x \in\left(I_{X}(b) \cap I_{X}^{\prime}(b)\right) \cap A_{\beta}$, then either $x \in X \backslash B$ hence $x \notin B$, or $x \notin B \backslash\left\{x_{\alpha}: \alpha \in \kappa\right\}$, hence $x=x_{\beta}$, a contradiction. Therefore $I_{X}(b) \cap I_{X}^{\prime}(b)$ intersects $\left\{A_{\alpha}: \alpha \in \kappa\right\}$ in $x_{\alpha}$. Now, if $b \in\left\{x_{\alpha}: \alpha \in \kappa\right\}$, then $b=x_{\alpha}$ for $\alpha \in \kappa$. Since, by construction, $U_{\alpha} \cap U_{\beta}=\varnothing$ for $\alpha \neq \beta$ and $A_{\beta} \subset U_{\beta}$, it follows that $U_{\alpha} \cap A_{\beta}=\varnothing$ for $\beta \neq \alpha$ except for $\beta=0$. Therefore $U_{\alpha}$ intersects finitely many elements of $\left\{A_{\alpha}: \alpha \in \kappa\right\}$.

Lemma 1.16. Let $X, Y$ be topological spaces and $B \subset X$ such that $\overline{X \backslash B} \cap B$ is discrete in $B$ and its points can be separated by a disjoint family of open sets in $X$. Then any $f: B \rightarrow Y$ continuous map can be extended to $X$, so that it remains continuous at points of $B$.

Proof. Let $\kappa$ be a cardinal with $|\overline{X \backslash B} \cap B|=\kappa$. By Lemma 1.15, there exists $\left\{A_{\alpha}: \alpha \leq \kappa\right\}$ a disjoint family of closed sets in $B$ such that $X=\left(\bigcup_{\alpha \in \kappa} A_{\alpha}\right) \cup B$ and $A_{\alpha} \cap B=\left\{x_{\alpha}\right\}$ for some $x_{\alpha}$. Let $g_{\alpha}: A_{\alpha} \rightarrow Y$ given by $g_{\alpha}(x)=f\left(x_{\alpha}\right)$. 
Then $g_{\alpha}$ 's are continuous maps. By Lemma 1.13, there exists $h=f \cup \bigcup_{\alpha \in \kappa} g_{\alpha}$, which extends $f$ to $X$ and is continuous at $B$.

Note that in the previous Lemma, if $|\overline{X \backslash B} \cap B|<\aleph_{0}$, we only need the space $X$ to be Hausdorff.

Corollary 1.17. Let $B \subseteq X$. If $\overline{X \backslash B} \cap B$ is locally discrete in $B$ and its points can be separated by a disjoint family of open sets in $X$, then there is a weak retraction of $X$ onto $B$.

Proof. Let $i d_{B}$ be the identity map on $B$. Apply Lemma 1.16 with $Y=B$.

With reference to Definition 1.5, the following proposition gives a characterization for a retraction of a subspace $Z$ of $X \times_{a d} 2$.

Proposition 1.18. Let $Z=(A \times\{1\}) \cup(B \times\{0\}) \subseteq X \times \times_{a d} 2$. Then $Z$ can be retracted onto $B \times\{0\}$ if and only if $A \cup B$ can be weakly retracted onto $B$.

Proof. Let $\phi$ be a retraction of $Z$ onto $B \times\{0\}$, and assume, without loss of generality, by Lemma 1.7, that for each $x \in A \cap B, \phi(x, 1)=\phi(x, 0)=(x, 0)$. For each $x \in A \cup B$ choose $i_{x} \in\{0,1\}$ such that $\left(x, i_{x}\right) \in Z$. Since $\phi(x, 1)=$ $\phi(x, 0)$ for each $x \in A \cup B$ the choice of $i_{x}$ does not matter. Define $f: A \cup B \rightarrow B$ by $f(x)=\iota^{-1} \circ \phi\left(x, i_{x}\right)$. For each $x \in B, f(x)=\iota^{-1} \circ \phi(x, 0)=x$. It remains to prove the continuity of $f$ at the points of $B$. Let $\left(x_{\sigma}\right)_{\sigma \in \Sigma}$ a net in $X, x \neq x_{\sigma}$ for each $\sigma \in \Sigma$ and $x \in B$, such that $x_{\sigma} \rightarrow x$. Choose $i_{\sigma} \in\{0,1\}$ where $\left(x_{\sigma}, i_{\sigma}\right) \in Z$. Then $\left(x_{\sigma}, i_{\sigma}\right) \rightarrow(x, 0)$. Since $\iota^{-1} \circ \phi$ is a continuous function, $\iota^{-1} \circ \phi\left(x_{\sigma}, i_{\sigma}\right) \rightarrow \iota \circ \phi(x, 0)$. Then $f\left(x_{\sigma}\right) \rightarrow f(x)$.

Vice versa, let $f: A \cup B \rightarrow B$ be a weak retraction and $(r \uparrow Z): Z \rightarrow$ $(A \cup B) \times\{0\}$ the retraction of $Z$ onto $B \times\{0\}$. Define $\phi: Z \rightarrow B \times\{0\}$ such that $\phi(x, i)=\iota \circ f \circ \iota^{-1} \circ(r \uparrow Z)(x, i)$. Then $\phi$ is a continuous map at the points of $B \times\{0\}$. Since $A \times\{1\}$ is a discrete subset of $X \times{ }_{a d} 2$, it follows that $\phi$ is continuous. Moreover $\phi\left\lceil B \times\{0\}=i d_{B \times\{0\}}\right.$.

Lemma 1.19. Let $X$ be a complete metrizable space, $Y \subseteq X$ such that $Y$ is a countable dense set with no isolated points. Then there is no weak retraction of $X$ onto $Y$.

Proof. By contradiction, let $f: X \rightarrow Y$ be a weak retraction and $A \subseteq X$ the set of all points in which $f$ is continuous. Then $Y \subseteq A$ and $A$ is a $G_{\delta}$ set in $X$, hence $A$ is a complete metrizable space. Observe that $A=\bigcup_{y \in Y}\left(f\lceil A)^{-1}(\{y\})\right.$, i.e., $A$ is an $F_{\sigma}$ in $X$. By Baire Category Theorem, there exists $y \in Y$ such that $(f \uparrow A)^{-1}(\{y\})$ is not nowhere dense, hence $(f \uparrow A)^{-1}(\{y\})$ contains $U$, an open set in $A$. Since $U \cap Y \neq\{y\}$, we have a contradiction.

Corollary 1.20. Let $Z=(\mathbb{P} \times\{1\}) \cup(\mathbb{Q} \times\{0\}) \subset[0,1] \times{ }_{a d} 2$. Then $Z$ cannot be retracted onto $\mathbb{Q} \times\{0\}$.

Proof. By Lemma 1.19, there is not a weakly retraction of $\mathbb{R}$ onto $\mathbb{Q}$. Proposition 1.18 ends the proof. 


\section{Properties preserved by the Alexandroff Duplicate and its SUBSPACES}

As a special case of the the Fundamental Theorem of Resolutions, we have that if $X$ is a compact space, then $X \times_{a d} 2$ is also compact.

Next we show that many properties of $X$ are preserved by its duplicate.

Lemma 2.1. $X$ is Tychonoff space if and only if $X \times_{a d} 2$ is Tychonoff.

Proof. First we show that $T_{1}$ is preserved. Any isolated point $(x, 1) \in X \times \times_{a d} 2$ is clopen. Moreover, since $X$ is $T_{1}$, and $X \cong X \times\{0\}$ is a closed subspace of $X \times_{a d} 2$, it follows that every point is closed in $X \times_{a d} 2$. Observe that for the points of $X \times\{1\}$, since they are isolated points, there exists always a continuous map $f: X \times_{a d} 2 \rightarrow I$ such that $f((x, 1))=0$ and $f((x, i))=1$ for any $(x, i) \neq(x, 1)$. Let $\left(x_{0}, 0\right) \in X \times_{a d} 2$ and $U^{*}=\hat{U} \backslash\left\{\left(x_{0}, 1\right)\right\}$ a neighborhood of $\left(x_{0}, 0\right)$. Since $X$ is Tychonoff, $x_{0} \in X$ and $U \in \tau_{X}$, there exists $g: X \rightarrow I$ continuous map such that $g\left(x_{0}\right)=0$ and $g\left(U^{c}\right)=1$. We define $f: X \times_{a d} 2 \rightarrow I$ such that $f((x, i))=1$ for $(x, i)=\left(x_{0}, 1\right)$ and $f((x, i)=g(x)$ otherwise. Then $f$ is continuous map such that $f\left(\left(x_{0}, 0\right)\right)=0$ and $f((x, i))=1$ for any $(x, i) \in$ $\left(X \times{ }_{a d} 2\right) \backslash U^{*}$.

Since $X \cong X \times\{0\}$, the vice versa holds as well.

Lemma 2.2. If $X$ is normal, then also $X \times_{a d} 2$ is normal.

Proof. Let $K$ and $C$ be two closed disjoint subsets of $X \times{ }_{a d} 2$. Let $K \cap X \times\{0\}=$ $K_{1}$ and $C \cap X \times\{0\}=C_{1}$ closed disjoint subsets in $X \times\{0\}$, by normality of $X$, there exists $U_{1}$ and $V_{1}$ open sets in $X \times\{0\}$ such that $K_{1} \subseteq U_{1}$ and $C_{1} \subseteq V_{1}$ and $U_{1} \cap V_{1}=\varnothing$. Then $V=\left(\hat{V}_{1} \backslash K\right) \cup(C \backslash X \times\{0\})$ and $U=\left(\hat{U}_{1} \backslash C\right) \cup(K \backslash X \times\{0\})$ are open disjoint subsets in $X \times_{a d} 2$ containing $C$ and $K$ respectively. Then $X \times_{a d} 2$ is normal.

Let $A$ and $B$ subsets of $X$ and $Z=(A \times\{1\}) \cap(B \times\{0\}) \subseteq X \times \times_{a d} 2$. Henceforth, unless we state otherwise, we denote a subset of $X \times_{a d} 2$ simply with $Z$.

In general a subspace $Z \subseteq X \times_{a d} 2$ need not to be normal even if $X$ is normal. Indeed, if $X$ is not hereditarily normal, there exists $A \subseteq X$ that is not normal. Take $Z=A \times\{0\}$. Then $Z$ is not normal as a subset of that normal space $X \times \times_{a d} 2$.

Lemma 2.3. Let $X$ be a normal space and $B$ a closed subset of $X$. Then $Z \subseteq X \times{ }_{a d} 2$ is normal.

Proof. Let $K=K^{\prime} \cap Z$ and $C=C^{\prime} \cap Z$ be two closed disjoint subsets of $Z$, with $K^{\prime}$ and $C^{\prime}$ closed subsets of $X \times{ }_{a d} 2$. Let $K \cap B \times\{0\}=K_{1}$ and $C \cap B \times\{0\}=C_{1}$ be closed disjoint subsets in $B \times\{0\}$. Since $B$ is a closed subset of $X$, and $B \times\{0\}$ is normal, there exists $U_{1}=U \cap B$ and $V_{1}=V \cap B$, with $U, V \in \tau$, which are disjoint open sets in $B \times\{0\}$ such that $K_{1} \subseteq U_{1}$ and $C_{1} \subseteq V_{1}$. Take $V^{*}=\left(\left(\hat{V}_{1} \cap Z\right) \backslash K\right) \cup(C \backslash B \times\{0\})$ and $U^{*}=\left(\left(\hat{U}_{1} \cap Z\right) \backslash C\right) \cup(K \backslash B \times\{0\})$. Then $V^{*}$ and $U^{*}$ are open disjoint subsets in $Z$ containing $C$ and $K$ respectively. 
Lemma 2.4. For any topological space $X$, the following hold:

(i) $l(X)=l\left(X \times_{a d} 2\right)$,

(ii) If $|X| \geq \aleph_{0}, c\left(X \times_{a d} 2\right)=|X|$,

(iii) $\chi(X)=\chi\left(X \times \times_{a d} 2\right)$.

Proof. (i). Observe that $l(X)=l(X \times\{0\}) \leq l\left(X \times{ }_{a d} 2\right)$, because $X \times\{0\}$ is closed. It is sufficient to show that $l\left(X \times \times_{a d} 2\right) \leq l(X)$. Let $\mathcal{U}$ be an open cover for $X \times_{a d} 2$, and assume, without loss of generality, that $\mathcal{U} \subseteq \mathcal{B}$. Thus, $\mathcal{U} \cap \mathcal{B}_{0}=\left\{\hat{U}_{i} \backslash\left(F_{U_{i}} \times\{1\}\right): i \in I \wedge F_{U_{i}} \in[X]^{<\omega}\right\}$. Since $l(X)=\kappa$, there exists an open refinement of $\left\{U_{i}\right\}_{i \in I},\left\{V_{i}\right\}_{i \in J}$ which cover $X$, such that $|J| \leq \kappa$. For each $V_{i} \in\left\{V_{i}\right\}_{i \in J}$ choose $U_{i} \in\left\{U_{i}\right\}_{i \in I}$, such that $V_{i} \subseteq U_{i}$, and define $F_{U_{i}}=F_{V_{i}}$. So $\left\{\hat{V}_{i} \backslash\left(F_{V_{i}} \times\{1\}\right): i \in J \wedge F_{V_{i}} \in[X]^{<\omega}\right\}$, leaves uncovered $\bigcup_{i \in J} F_{V_{i}} \times\{1\}$ which can be covered by $|J|$ many open sets. Thus $l\left(X \times{ }_{a d} 2\right) \leq \kappa$.

(ii). Let $|X|=\kappa \geq \aleph_{0}$. The set $X \times\{1\} \subset X \times_{a d} 2$ is a set of isolated points of size $\kappa$, so $c(X \times\{1\})=\kappa$. Since $X \times\{1\}$ is an open subset of $X \times \times_{a d} 2$, $c(X \times\{1\}) \leq c\left(X \times_{a d} 2\right)$. Thus $c\left(X \times \times_{a d} 2\right) \geq \kappa$. From $c(X \times\{0\}) \leq\left|X \times_{a d} 2\right|$, if follows that $c\left(X \times \times_{a d} 2\right) \leq \kappa$.

(iii). For each $a=(x, 1) \in X \times\{1\},\{a\}$ is a local base at $a$. Thus $\chi(a, X \times a d$ $2)=1$. Next, let $a=(x, 0) \in X \times\{0\}$ and $\chi(X)=\mathrm{k}$. Let $\mathcal{U}(x)$ be a local base at each $x \in X$. Then $\{\hat{U} \backslash\{(x, 1)\}: U \in \mathcal{U}(x)\}$ is a local base at $a=(x, 0) \in X \times\{0\}$, which has the same size as $\mathcal{U}(x)$. Then $\chi(X \times\{0\})=$ $\chi\left(X \times_{a d} 2\right)=\chi(X)$.

Lemma 2.5. If $\mathcal{U}$ is a locally finite family in $X$, then $\hat{\mathcal{U}}$ is a locally finite family in $X \times \times_{a d} 2$.

Proof. Let $z=(x, i) \in X \times \times_{a d} 2$, observe that if $V$ is a neighborhood of $x$ in $X$, then $\hat{V}$ is a neighborhood of $z$ in $X \times_{a d} 2$. Now, since $\mathcal{U}$ is a locally finite family of $X$, there exists a neighborhood $V_{x}$ of $x$ which intersects only finitely many elements of $\mathcal{U}$. Thus $\hat{V}_{x}$ is a neighborhood of $z$ in $X \times_{a d} 2$, which intersect only finitely many elements of $\hat{\mathcal{U}}$.

Remark 2.6. Furthermore, if $\mathcal{U}$ is a locally finite family of open subsets of $X$, for any map $f: \mathcal{U} \rightarrow[\mathrm{X} \times 2]^{<\omega}$ such that $U \rightarrow F_{U}$, the set $\left\{\hat{U} \backslash F_{U}: U \in \mathcal{U}\right\}$ is locally finite family of open subsets of $X \times{ }_{a d} 2$.

Proposition 2.7. If $X$ is a paracompact space, then also $X \times_{a d} 2$ is a paracompact space.

Proof. Let $\mathcal{U}$ be an open cover for $X \times_{a d} 2$, and assume, without loss of generality, $\mathcal{U} \subset \mathcal{B}$. So $\mathcal{U} \cap \mathcal{B}_{0}=\left\{\hat{U} \backslash\left(F_{U} \times\{1\}\right): U \in \mathcal{U}^{\prime} \wedge F_{U} \in[X]^{<\omega}\right\}$ where $\mathcal{U}^{\prime}$ is a cover of $X$. By paracompactness of $X$, there exist an open refinement $\mathcal{V}^{\prime}$ of $\mathcal{U}^{\prime}$ which is locally finite. For each $V \in \mathcal{V}^{\prime}$, choose $U \in \mathcal{U}^{\prime}$ such that $V \subseteq U$. Define $F_{V}=F_{U}$. Then $\mathcal{V}_{0}=\left\{\hat{V} \backslash F_{V} \times\{1\}: F_{V} \in[X]^{<\omega}, V \in \mathcal{V}^{\prime}\right\}$ is also a locally finite family of $X \times_{a d} 2$ and it is an open refinement of $\mathcal{U} \cap \mathcal{B}_{0}$. Let $\mathcal{V}_{1}=\left\{\{(x, 1)\}:(x, 1) \notin \cup \mathcal{V}_{0}\right\}$. Then $\mathcal{V}_{1}$ is a discrete family of open sets, since $X \times\{0\} \subseteq \cup \mathcal{V}_{0}$. Thus $\mathcal{V}=\mathcal{V}_{0} \cup \mathcal{V}_{1}$ is an open refinement of $\mathcal{U}$ which is locally finite. 
Proposition 2.8. If $X$ is an hereditarily paracompact space, then also $X \times_{a d} 2$ is an hereditarily paracompact space.

Proof. It is sufficient to show that any open subspace of $X \times_{a d} 2$ is paracompact (see [4]). Let $A$ be an open subspace of $X \times_{a d} 2$ and $\mathcal{U}$ an open cover of $A$, without loss of generality, assume that $\mathcal{U} \subset \mathcal{B}$. Then $\mathcal{U}=\mathcal{U}_{0} \cup \mathcal{U}_{1}$ where $\mathcal{U}_{0}=\left\{\hat{U} \backslash\left(F_{U} \times\{1\}\right): U \in \mathcal{U}^{\prime} \wedge F_{U} \in[X]^{<\omega}\right\}$ and $\mathcal{U}^{\prime}$ is an open covering of $\pi_{0}(A \cap X \times\{0\})$, and $\mathcal{U}_{1} \subseteq\{\{(x, 1)\}:(x, 1) \in A\}$. Since $X$ is hereditarily paracompact, there exists $\mathcal{V}^{\prime}$ open refinement of $\mathcal{U}^{\prime}$ which is locally finite at the points of $\pi_{0}(A \cap X \times\{0\})$. Now, for each $V \in \mathcal{V}^{\prime}$, choose $U \in \mathcal{U}^{\prime}$ such that $V \subseteq U$ and define $F_{V}=F_{U}$. Let $\mathcal{V}_{0}=\left\{\hat{V} \backslash\left(F_{V} \times\{1\}\right): V \in \mathcal{V}^{\prime}\right\}$ and $\mathcal{V}_{1}=\left\{\{(x, 1)\}:(x, 1) \notin \cup \mathcal{V}_{0}\right\} \cap \mathcal{U}_{1}$. Then $\mathcal{V}_{1}$ is a discrete family of open sets and $\mathcal{V}=\mathcal{V}_{0} \cup \mathcal{V}_{1}$ is an open refinement of $\mathcal{U}$ which is locally finite. Then $A$ is paracompact in $X \times{ }_{a d} 2$.

Corollary 2.9 (Alexandroff, Urysohn [1]). [0,1] $\times_{\text {ad }} 2$ is a first countable compact Hausdorff, hereditarily paracompact space which has an uncountable disjoint family of open sets.

From the definition of Menger-Urysohn dimension, it follows that ind $X \leq n$ with $n \geq 0$ if and only if there exists a base $\mathcal{B}$ of $X$ such that ind $\partial B \leq n-1$ for each $B \in \mathcal{B}$. In particular ind $X=0$ if and only if the space is 0 -dimensional and for every subspace $Y \subset X$, and we have that ind $Y \leq \operatorname{ind} X$.

In order to calculate the small inductive dimension of the Alexandroff duplicate and its subspaces, we need the following lemmas.

Lemma 2.10. Let $(X, \tau)$ be a topological space, and $U, V \in \tau$. Then

(i) $\overline{\hat{V}} \subseteq \hat{\bar{V}}$ and $\hat{\bar{V}} \backslash \overline{\hat{V}}=\partial V \times\{1\}$

(ii) If $\bar{V} \subseteq U$, then $\overline{\hat{V}} \subseteq \hat{U}$

Proof. Observe that $\overline{V \times\{0\}}=\bar{V} \times\{0\}$ and $\overline{V \times\{1\}}=V \times\{1\} \cup V^{\prime} \times\{0\}$. Then $\overline{\hat{V}}=\overline{V \times\{0\}} \cup \overline{V \times\{1\}}=\overline{V \times\{0\}} \cup V \times\{1\} \subseteq \overline{V \times\{0\}} \cup \overline{V \times\{1\}}=\overline{\bar{V}}$. Moreover $\hat{\bar{V}} \backslash \overline{\hat{V}}=(\bar{V} \times\{0\} \cup \bar{V} \times\{1\}) \backslash(\bar{V} \times\{0\} \cup \overline{V \times\{1\}})=\bar{V} \times\{1\} \backslash V \times\{1\}=$ $\partial V \times\{1\}$.

If $\bar{V} \subseteq U$, by (i), $\overline{\hat{V}} \subseteq \hat{\bar{V}} \subseteq \hat{U}$.

Lemma 2.11. Let $Z \subseteq X \times_{a d} 2$. If $W \subseteq Z$, then

$$
c l_{Z}(W) \cap(A \times\{1\})=W \cap(A \times\{1\})=\operatorname{int}_{Z}(W) \cap(A \times\{1\})
$$

Proof. For each $(x, 1) \in A \times\{1\},(x, 1) \in \operatorname{cl}_{Z}(W)$ if and only if $(x, 1) \in W$, and $(x, 1) \in \operatorname{int}_{Z}(W)$ if and only if $(x, 1) \in W$.

Lemma 2.12. Let $Z \subseteq X \times_{a d} 2$ and $D \subseteq B$. Then

(i) $\operatorname{cl}_{Z}(\hat{D}) \cap(B \times\{0\})=\operatorname{cl}_{B}(D) \times\{0\}$;

(ii) $\operatorname{int}_{Z}(\hat{D}) \cap(B \times\{0\})=\operatorname{int}_{B}(D) \times\{0\}$;

(iii) $\partial_{Z}(\hat{D} \cap(A \times\{1\}))=((D \cap A) \times\{0\})^{\prime}$;

(iv) $c l_{Z}(\hat{D} \cap Z)=\left(c_{B}(D) \times\{0\}\right) \cup(D \cap(A \times\{1\}))$; 
(v) $\operatorname{int}_{Z}(\hat{D} \cap Z)=\left(\operatorname{int}_{B}(D) \times\{0\}\right) \cup(D \cap(A \times\{1\}))$;

(vi) $\partial_{B \times\{0\}}(D \times\{0\})=\partial_{Z}(\hat{D} \cap Z)$.

Proof. (i). The point $(x, 0)$ belongs to $\operatorname{cl}_{Z}(\hat{D}) \cap(B \times\{0\})$ if and only if for each $U^{*} \in \tau_{Z}(x, 0), U^{*}=(\hat{U} \backslash\{(x, 1)\}) \cap Z, U^{*} \cap(\hat{D}) \neq \varnothing$ hence $U^{*} \cap(D \times\{0\}) \neq \varnothing$. Thus $(x, 0) \in \operatorname{cl}_{Z}(D \times\{0\}) \cap B \times\{0\}=\operatorname{cl}_{B}(D) \times\{0\}$.

(ii). Clearly if $(x, 0) \in \operatorname{int}_{X}(D \cap B) \times\{0\}=\operatorname{int}_{B}(D) \times\{0\}$, then $(x, 0) \in$ $\operatorname{int}_{Z}(\hat{D})$. Moreover, if $(x, 0) \in \operatorname{int}_{Z}(\hat{D})$, there exists $U^{*} \in \tau_{Z}(x, 0), U^{*}=$ $(\hat{U} \backslash\{(x, 1)\}) \cap Z, U^{*} \subseteq \hat{D}$, so $(U \cap B) \times\{0\} \subseteq D \times\{0\}$. Hence $(x, 0) \in$ int $_{X}((D \cap B) \times\{0\})=\operatorname{int}_{B}(D) \times\{0\}$.

(iii). Since $\hat{D} \cap(A \times\{1\})$ is an open subset of $Z, \partial_{Z}(\hat{D} \cap A \times\{1\})=$ $\hat{D} \cap(A \times\{1\}) \backslash(\hat{D} \cap(A \times\{1\}))$. Moreover, for each $(x, 1) \in Z \backslash(\hat{D} \cap A \times$ $\{1\}),(x, 1) \notin \overline{\hat{D} \cap(A \times\{1\})}$, thus for each $(x, 1) \in Z \backslash(\hat{D} \cap(A \times\{1\}))$, $(x, 1) \notin \partial_{Z}(\hat{D} \cap(A \times\{1\}))$. For each $(x, 0) \in Z \backslash(\hat{D} \cap(A \times\{1\})),(x, 0) \in$ $\overline{(\hat{D} \cap(A \times\{1\}))}$ if and only if $(x, 0) \in(D \cap A) \times\{1\} \cup((D \cap A) \times\{0\})^{\prime}$, since $\overline{(\hat{D} \cap(A \times\{1\}))}=(D \cap A) \times\{1\} \cup((D \cap A) \times\{0\})^{\prime}$. Then $\partial_{Z}(\hat{D} \cap(A \times\{1\}))=$ $((D \cap A) \times\{0\})^{\prime}$.

(iv). From (i), it follows that $\operatorname{cl}_{Z}(\hat{D} \cap Z) \subseteq \operatorname{cl}_{B}(D) \times\{0\} \cup(D \cap(A \times\{1\}))$. On the other hand, $\operatorname{cl}_{Z}(\hat{D} \cap Z) \supseteq \operatorname{cl}_{Z}(D \times\{0\})=\operatorname{cl}_{B}(D) \times\{0\}$ and $\operatorname{cl}_{Z}(\hat{D} \cap Z) \supseteq$ $(\hat{D} \cap Z) \supseteq(D \cap(A \times\{1\}))$.

(v). From (ii), it follows that $\operatorname{int}_{Z}(\hat{D} \cap Z) \subseteq \operatorname{int}_{B}(D) \times\{0\} \cup(D \cap(A \times\{1\}))$. On the other hand, we have that $\operatorname{int}_{Z}(\hat{D} \cap Z) \supseteq \operatorname{int}_{Z}(\hat{D} \cap Z) \cap(A \times\{1\})=$ $(D \cap A) \times\{1\}$ and $\operatorname{int}_{Z}(\hat{D} \cap Z) \supseteq \operatorname{int}_{Z}\left(\widehat{\operatorname{int}_{B}} \cap Z\right)=\widehat{\operatorname{int}_{B}} \cap Z \subseteq\left(\widehat{\operatorname{int}_{B}} \cap Z\right) \cap(B \times\{0\})$ $=\operatorname{int}_{B}(D) \times\{0\}$.

Theorem 2.13. Let $Z \subseteq X \times_{a d} 2$ with $B \neq \varnothing$, then ind $Z=$ indB.

Proof. Since $B \simeq B \times\{0\} \subset Z$, then $\operatorname{ind} B \leq \operatorname{ind} Z$. It is sufficient to show that $\operatorname{ind} Z \leq \operatorname{ind} B$, using induction on $\operatorname{ind} B$.

For ind $B=0$, there exists a base $\mathcal{G}$ for $B$, such that for each $G \in \mathcal{G}, G$ is clopen in $B$, then for each $G \in \mathcal{G}$, also $\hat{G} \cap Z$ is clopen in Z. Now, for each $G \in \mathcal{G}$ and $F \in[A]^{<\omega},(\hat{G} \backslash F \times\{1\}) \cap Z$ is a basic open sets in $Z$. They are also a clopen sets, because the boundaries never contain isolated points. Then $\{(a, 1)\}_{a \in A} \cup\left\{(\hat{G} \backslash F \times\{1\}) \cap Z: F \in[A]^{<\omega}, G \in \mathcal{G}\right\}$ is a base of clopen sets for $Z$ and ind $Z=0$.

Let $n \in \omega$ and $\operatorname{ind} B=n$, we want to prove that $\operatorname{ind} Z \leq n$. Since $\operatorname{ind} B=n$ there exists a base $\mathcal{G}$ for $B$, such that for each $G \in \mathcal{G}$, ind $\partial_{B} G \leq n-1$. Thus $\partial_{B \times\{0\}} G \times\{0\} \leq n-1$. Let $(\hat{G} \backslash F \times\{1\}) \cap Z$ the corresponding basic open set in $Z$, then from Lemma 2.12 we have that $\partial_{Z}((\hat{G} \backslash F \times\{1\}) \cap Z)=$ $\partial_{Z}(\hat{G} \cap Z)=\partial_{B \times\{0\}} G \times\{0\}$, then it follows that ind $\partial_{Z}((\hat{G} \backslash F \times\{1\}) \cap Z)=$ ind $\partial_{B \times\{0\}} G \times\{0\} \leq n-1$. Thus ind $Z \leq n-1$. 
We conclude this section analyzing the Baire property of the Alexandroff duplicate and its subspaces. We first characterize dense sets of subspaces of the Alexandroff duplicate.

Lemma 2.14. A topological space $(X, \tau)$ has a dense set of isolated points, if and only if any $\left\{D_{i}\right\}_{i \in I}$ arbitrary family of dense subsets of $X$ is such that $\bigcap_{i \in I} D_{i}$ is dense too.

Proof. Let $D$ a dense set in $X$ consisting of isolated points. Notice that if $D_{i}$ is a dense set in $X$, then $D \subseteq D_{i}$, otherwise there exist $x \in D \backslash D_{i}$ and $\{x\}$ is an open set which does not intersect $D_{i}$, contradiction. Therefore $D \subseteq \bigcap_{i \in I} D_{i}$. Viceversa, if $X$ does not have a dense set of isolated points, then there exists an open set $U$ such that $U$ has no isolated point. Now, for each $x \in U, X \backslash\{x\}$ is dense, so $\bigcap_{x \in U} X \backslash\{x\}$ is dense too. We have a contradiction, because $X \backslash U=\bigcap_{x \in U} X \backslash\{x\}$ is not dense in $X$.

Lemma 2.15. Let $(X, \tau)$ be a topological space. If $\left\{D_{i}\right\}_{i \in I}$ is an arbitrary family of dense subsets of $X \times_{a d} 2$, then $\bigcap_{i \in I} D_{i}$ is dense too.

Proof. The set $X \times\{1\} \subseteq X \times_{a d} 2$ is a dense set of isolated points. The result follows from Lemma 2.14 .

Corollary 2.16. $X \times_{a d} 2$ is Baire for each topological space $X$.

Lemma 2.17. Let $Z \subseteq X \times_{a d} 2$. An open set $U$ is a dense subset of $Z$ if and only if there exists $D$ dense open in $B \backslash A^{\prime}$ and $U=(A \times\{1\}) \cup(D \times\{0\})$.

Proof. Let $U=(A \times\{1\}) \cup(D \times\{0\})$ be a subset of $Z$. Since $D$ is dense in $B \backslash A^{\prime}$, we have $\overline{D \times\{0\}}=\bar{D} \times\{0\}=\left(B \backslash A^{\prime}\right) \times\{0\}$. Then $\bar{U}=$ $\overline{(A \times\{1\}) \cup(D \times\{0\})}=(A \times\{1\}) \cup \overline{D \times\{0\}}=A \times\{1\} \cup\left(B \cap A^{\prime}\right) \times\{0\} \cup(B \backslash$ $\left.A^{\prime}\right) \times\{0\}=Z$. Since $B \backslash A^{\prime}$ is an open set we have that $U$ is an open set in $Z$.

Let $U$ be a dense open set in $Z$, then $A \times\{1\} \subseteq U$. Assume by contradiction that for every dense open in $B \backslash A^{\prime}$ we have that either $U \subsetneq(A \times\{1\}) \cup(D \times\{0\})$ or $U \supsetneq(A \times\{1\}) \cup(D \times\{0\})$. Let $D=U \cap\left(B \backslash A^{\prime}\right)$. Since $\left(B \backslash A^{\prime}\right) \times\{0\}$ is open in $Z$, i.e., $D$ is dense open in $B \backslash A^{\prime}$. Then $V=(A \times\{1\}) \cup(D \times\{0\})$ is dense open set in $Z$ such that $V \subseteq U$. If $U \supsetneq(A \times\{1\}) \cup(D \times\{0\})$ then $V \supsetneq U$ which is a contradiction. If $U \subsetneq(A \times\{1\}) \cup(D \times\{0\})$ we have that $U \subsetneq V \subseteq U$, a contradiction.

Lemma 2.18. Let $\left\{D_{\alpha}\right\}_{\alpha \in \kappa}$ be a family of dense open sets in $B \backslash A^{\prime}$. Then $\bigcap_{\alpha \in \kappa} A \times\{1\} \cup D_{\alpha} \times\{0\}$ is dense in $Z$ if and only if $\bigcap_{\alpha \in \kappa} D_{\alpha}$ is dense in $B \backslash A^{\prime}$.

Proof. $\overline{\bigcap_{\alpha \in \kappa}(A \times\{1\}) \cup\left(D_{\alpha} \times\{0\}\right)}=Z$ if and only if $\overline{A \times\{1\}} \cup \overline{\bigcap_{\alpha \in \kappa} D_{\alpha} \times\{0\}} \supseteq$ $Z$ if and only if $A \times\{1\} \cup\left(B \cap A^{\prime}\right) \times\{0\} \cup \overline{\bigcap_{\alpha \in \kappa} D_{\alpha} \times\{0\}} \supseteq Z$ if and only if $\overline{\bigcap_{\alpha \in \kappa} D_{\alpha} \times\{0\}} \supseteq\left(B \backslash A^{\prime}\right) \times\{0\}$, i.e., $\bigcap_{\alpha \in \kappa} D_{\alpha}$ is dense in $B \backslash A^{\prime}$.

Definition 2.19. A topological space $X$ is $\kappa$-Baire if the intersection of less then $\kappa$ dense open sets is dense; in this case, we write Baire $(X)=\kappa$. Further, 
we set $\operatorname{Baire}(X)=\infty$ if for all dense open sets their intersection is dense, and assume that $\operatorname{Baire}(\varnothing)=\infty$.

Theorem 2.20. Let $Z \subseteq X \times_{a d} 2$. Then $\operatorname{Baire}(Z)=\operatorname{Baire}\left(B \backslash A^{\prime}\right)$.

Proof. To prove the equality, we first observe that if $\left(B \backslash A^{\prime}\right) \times\{0\}=\varnothing$, then $A^{\prime} \supseteq B$, i.e., $\overline{A \times\{1\}}=Z$. Thus $Z$ has a dense set of isolated points and so Baire $(Z)=\operatorname{Baire}\left(\left(B \backslash A^{\prime}\right) \times\{0\}\right)=\infty$. Assume that $\left(B \backslash A^{\prime}\right) \times\{0\} \neq$ $\varnothing$ and $\operatorname{Baire}(Z)=\kappa$. Since $Z$ has $\kappa$ many dense open sets with non-dense intersection, by Lemma 2.17, without loss of generality, dense open sets have form $A \times\{1\} \cup D_{\alpha}$ with $\alpha \in \kappa$, and $D_{\alpha}$ 's are dense open sets in $\left(B \backslash A^{\prime}\right) \times\{0\}$. By Lemma 2.17, $\bigcap_{\alpha \in k} D_{\alpha} \times\{0\}$ is not dense in $\left(B \backslash A^{\prime}\right) \times\{0\}$. So $\left(B \backslash A^{\prime}\right) \times\{0\}$ has $\kappa$ many dense open sets with non-dense intersection. Viceversa, assume that Baire $\left(\left(B \backslash A^{\prime}\right) \times\{0\}\right)=\kappa$, so $B \backslash A^{\prime}$ has $\kappa$ many dense open sets $\left\{D_{\alpha}\right\}_{\alpha \in k}$ with non-dense intersection. By Lemma 2.17 and Lemma 2.18, $\left\{A \times\{1\} \cup D_{\alpha} \times\right.$ $\{0\}\}_{\alpha \in \kappa}$ is a family of dense open sets with non-dense intersection. Thus $Z$ has $\kappa$ many dense open sets with non-dense intersection, i.e., $\operatorname{Baire}(Z) \leq \kappa$.

\section{Metrizability AND LindelÖF PROPERTY}

Before extracting the Michael line and its relatives from $[0,1] \times_{a d} 2$, we prove some useful characterizations of those subspaces of $X \times_{a d} 2$ that are metrizable or Lindelöf.

Definition 3.1. Let $(X, \tau)$ be a topological space and $A$ and $B$ subsets of $X$. $A$ is $\kappa$-discrete in $A \cup B$, if $A$ is the union of $\kappa$ many sets with no accumulation points in $B$.

Lemma 3.2. Let $Z \subseteq X \times_{a d} 2$. If every closed set of $Z$ is $G_{\kappa}$, then $A$ is $\kappa$-discrete in $A \cup B$.

Proof. Since $A \times\{1\}$ is open set in $Z$, then it is an $F_{\kappa}$ set. Let $A=\cup\left\{K_{\alpha}\right.$ : $\alpha \in \kappa\}$ where each $K_{\alpha} \times\{1\}$ is closed in $Z$. By contradiction, if $K_{\alpha}$ had an accumulation point $b \in B$, then $K_{\alpha} \times\{1\}$ would have an accumulation point at $(b, 0) \in Z$.

Lemma 3.3. Let $Z \subseteq X \times_{a d} 2$ and $K \subseteq A$ such that $K$ has no accumulation points in $B$. Then $K \times\{1\}$ is closed in $\bar{Z}$.

Proof. Since $K$ has no accumulation points in $B$, for each $b \in B$ there exists $U \in \tau_{X}(b)$ such that $U \cap K \subseteq\{b\}$. For each $(b, 0) \in B \times\{0\}$ there exists $U_{Z}=(\hat{U} \backslash\{(b, 1)\}) \cap Z$ such that $U_{Z} \cap(K \times\{1\})=\varnothing$. Then $K \times\{1\}$ has no accumulation points in $B \times\{0\}$. On the other hand, since every point in $A \times\{1\}$ is an isolated point, $K \times\{1\}$ cannot have accumulation points in $A \times\{1\}$. Thus $K \times\{1\}$ has no accumulation points, so $K \times\{1\}$ is closed in $Z$.

Definition 3.4. Let $(X, \tau)$ be a topological space and $A \subseteq X$. We say that $\mathcal{B}$ is a base for the points of $A$ in $X$, if each $B \in \mathcal{B}$ is an open set in $X$ and for each $x \in A$ and $U \in \tau_{X}(x)$ there exist $B \in \mathcal{B}$ such that $x \in B \subseteq U$. 
Theorem 3.5. Let $Z \subseteq X \times_{a d} 2$ and $\kappa$ be an infinite cardinal. Then $Z$ has a $\kappa$-discrete base if and only if $B$ has a $\kappa$-discrete base in $X$ and $A$ is $\kappa$-discrete in $A \cup B$.

Furthermore we have:

(i) If $\mathcal{B}$ is a $\kappa$-discrete base for $B$ in $X$ and $A=\bigcup_{\beta<\kappa} K_{\beta}$ with each $K_{\beta}$ having no accumulation points in $B$, then $\mathcal{B}^{*}=\left\{\hat{V} \backslash\left(\bigcup_{\beta \in F} K_{\beta} \times\{1\}\right)\right.$ : $\left.F \in[\kappa]^{<\omega}, V \in \mathcal{B}\right\}$ is a $\kappa$-discrete base for $B \times\{0\}$ in $Z$.

(ii) If $\mathcal{B}^{*}=\bigcup_{\gamma \in \kappa} \mathcal{B}_{\gamma}{ }^{*}$ is a $\kappa$ discrete base for $Z$, then $\mathcal{B}_{B \times\{0\}}=\bigcup_{\gamma \in \kappa}\{U \cap$ $\left.(B \times\{0\}): U \in \mathcal{B}_{\gamma}^{*}\right\}$ is a $\kappa$-discrete base for $B \times\{0\}$ in $Z$ and $A=$ $\bigcup_{\gamma \in \kappa}\left\{a:\{(a, 1)\} \in \mathcal{B}_{\gamma}^{*}\right\}$.

Proof. Assume that $\mathcal{B}$ is a $\kappa$-discrete base for $B$ in $X$ and $A=\bigcup_{\alpha \in \kappa} K_{\alpha}$ where each $K_{\alpha}$ has no accumulation points in $B$. Then $\mathcal{K}_{\alpha}=\left\{\{(k, 1)\}: k \in K_{\alpha}\right\}$ is a discrete family of open sets in $Z$ and $\mathcal{K}=\cup\left\{\mathcal{K}_{\alpha}: \alpha \in \kappa\right\}$ is a $\kappa$-discrete open family which is a base for $A \times\{1\}$.

Next we want to find a $\kappa$-discrete base for $B \times\{0\}$ in $Z$. Let $(b, 0) \in B \times\{0\}$, and $(\hat{U} \backslash\{(b, 1)\}) \cap Z$ a neighborhood of $(b, 0)$ in $Z$. If $b \in A$, there exists $\gamma \in \kappa$ such that $(b, 1) \in K_{\gamma} \times\{1\}$ which is closed in $Z$ by Lemma 3.3. Moreover there exists $V \in \mathcal{B}$ such that $b \in V \subseteq U$. Let $F=\{\gamma\}$ hence $(b, 0) \in \hat{V} \backslash\left(\bigcup_{\beta \in F} K_{\beta} \times\right.$ $\{1\}) \cap Z \subseteq \hat{V} \backslash\{(b, 1)\} \cap Z \subseteq \hat{U} \backslash\{(b, 1)\} \cap Z$. Then $\mathcal{B}^{*}$ is a base for $B \times\{0\}$ in $Z$. Since $\mathcal{B}$ is a $\kappa$-discrete base for $B$, we have that $\mathcal{B}=\bigcup_{\gamma \in \kappa} \mathcal{B}_{\gamma}$. Then $\mathcal{B}^{*}=\bigcup_{\gamma \in \kappa} \mathcal{B}_{\gamma}{ }^{*}$ where $\mathcal{B}_{\gamma}{ }^{*}=\left\{\hat{V} \backslash\left(\bigcup_{\beta \in F} K_{\beta} \times\{1\}\right): F \in[\kappa]^{<\omega}, V \in \mathcal{B}_{\gamma}\right\}$. It remains to prove that for each $\gamma \in \kappa, \mathcal{B}_{\gamma}{ }^{*}$ is a $\kappa$-discrete family in $Z$. Since for every $\gamma \in \kappa, \mathcal{B}_{\gamma}$ is discrete family, then $\left\{\hat{V}: V \in \mathcal{B}_{\gamma}\right\}$ is discrete too. Let $\gamma \in \kappa$ and $F \in[\kappa]^{<\omega}$ be fixed, then $\left\{\hat{V} \backslash\left(\bigcup_{\xi \in F} K_{\xi} \times\{1\}\right): V \in \mathcal{B}_{\gamma}\right\} \subseteq\left\{\hat{V}: V \in \mathcal{B}_{\gamma}\right\}$ is a discrete family. Hence $\mathcal{B}^{*}=\bigcup_{\gamma \in \kappa}\left(\bigcup\left\{\hat{V} \backslash\left(\bigcup_{\xi \in F} K_{\xi} \times\{1\}\right): F \in[\kappa]^{<\omega}, V \in\right.\right.$ $\left.\left.\mathcal{B}_{\gamma}\right\}\right)$ is $\kappa$-discrete too. Further, $\mathcal{B}^{*} \cup \mathcal{K}$ is a $\kappa$-discrete base for $Z$.

Assume now that $Z$ has a $\kappa$-discrete base $\mathcal{B}^{*}=\bigcup_{\gamma \in \kappa} \mathcal{B}_{\gamma}^{*}$. Set $\mathcal{B}_{B \times\{0\}}=$ $\bigcup_{\gamma \in \kappa}\left\{U \cap(B \times\{0\}): U \in \mathcal{B}_{\gamma}^{*}\right\}$. Then $\mathcal{B}_{B \times\{0\}}$ is a $\kappa$-discrete base for $B \times\{0\}$ in $Z$. Since $A \times\{1\}$ is a set of isolated points in $Z$, then for each $a \in A$, the set $\{(a, 1)\} \in \mathcal{B}^{*}$. Hence $K_{\gamma}=\left\{a:\{(a, 1)\} \in \mathcal{B}_{\gamma}^{*}\right\}$ is a discrete family of points such that $A=\bigcup_{\gamma<\kappa} K_{\gamma}$. Clearly every $K_{\gamma}$ has no accumulation points in $B$, otherwise $K_{\gamma}$ would not be discrete.

Definition 3.6. Let $(X, \tau)$ be a regular topological space and $A \subseteq X$. We say that $A$ is metrizable in $X$ if there exists a $\sigma$-discrete open family in $X$ which is a base for the points of $A$ in $X$.

Corollary 3.7. Let $X$ be a regular space. The subspace $Z \subseteq X \times_{a d} 2$ is metrizable if and only if $B$ has a countable base in $X$, and $A$ is the union of countable many sets with no accumulation points in $B$.

Proof. Since $X \times_{a d} 2$ is regular, it follows from Theorem 3.5.

In the following we characterize the Lindelöf property of a subspace $Z \subseteq$ $X \times_{a d} 2$. First we need the following lemma. 
Lemma 3.8. If $Z \subseteq X \times_{a d} 2$ is Lindelöf, then every uncountable subset $K$ of $A$, with $\mathrm{cf}|\mathrm{K}|>\aleph_{0}$, has a complete accumulation point in $B$.

Proof. Assume that $Z$ is Lindelöf . By contradiction, assume that there exists $K \subset A$ such that $\mathrm{cf}|\mathrm{K}|>\aleph_{0}$ and for each $b \in B$ there exists $U_{b} \in \tau_{X}$ containing $b$ such that $\left|U_{b} \cap K\right|<|K|$. Let $U_{b}{ }^{*}=\hat{U}_{b} \backslash\{(b, 1)\} \cap Z$ the corresponding open set in $Z$. Then $\left\{U_{b}{ }^{*}\right\}_{b \in B}$ is an open cover of $B \times\{0\}$, which is a closed subset of a Lindelöf space. By Lindelöfness of $B \times\{0\}$ we can find a countable open subcover $\left\{U_{b_{i}}{ }^{*}\right\}_{i \in \omega}$ of $B \times\{0\}$. Let $U^{*}=\bigcup_{i \in \omega} U_{b_{i}}{ }^{*}$. Then $B \times\{0\} \subset U^{*}$ and

$$
\left|U^{*} \cap K \times\{1\}\right|=\left|\bigcup_{i \in \omega}\left(U_{b_{i}}{ }^{*} \cap K \times\{1\}\right)\right| \leq \Sigma_{i \in \omega}\left|U_{b_{i}}{ }^{*} \cap K \times\{1\}\right| .
$$

Since $\left|U_{b_{i}}{ }^{*} \cap K \times\{1\}\right|<|K|$ and $\mathrm{cf}|\mathrm{K}|>\aleph_{0}$, it follows that $\left|U^{*} \cap K \times\{1\}\right|<|K|$. Now, denote $\tilde{A}=\left\{a \in K:(a, 1) \notin U^{*}\right\}$ and let $\mathcal{U}=\left\{U^{*}\right\} \cup\{(a, 1): a \in \tilde{A}\}$ be a cover of $Z$. Since $\{(a, 1): a \in \tilde{A}\}$ contain an uncountable subset of isolated points we cannot find a countable subcover of $\mathcal{U}$ in contradiction with the Lindelöfness of $Z$.

Theorem 3.9. Let $Z \subseteq X \times_{a d}$ 2. The following statements are equivalent:

(i) $Z$ is Lindelöf;

(ii) $B$ is Lindelöf and every uncountable subset $K$ of $A$, with $\mathrm{cf}|\mathrm{K}|>\aleph_{0}$, has a complete accumulation point in $B$;

(iii) $B$ is Lindelöf and every uncountable subset $K$ of $A$, with $|K|$ regular cardinal has a complete accumulation point in $B$;

(iv) $B$ is Lindelöf and every uncountable subset $K$ of $A$, with $|K|=\aleph_{1}$ has a complete accumulation point in $B$;

(v) $B$ is Lindelöf and every uncountable subset $K$ of $A$ has an accumulation point in $B$;

(vi) $B$ is Lindelöf and for every open subset $U$ in $X$ containing $B$, we have $|A \backslash U| \leq \aleph_{0}$

(vii) $B$ is Lindelöf and for every closed subset $F$ in $X$ that misses $B$, we have $|A \cap F| \leq \aleph_{0}$.

Proof. Assume that (i) holds. Since $B \times\{0\}$ is closed subspace of $Z$, it follows that $B \times\{0\}$ and thus $B$ is Lindelöf. Hence (ii) follows from Lemma 3.8.

The implications (ii) $\Rightarrow$ (iii) and (iii) $\Rightarrow$ (iv) are immediate.

For (iv) $\Rightarrow$ (v), let $K$ be uncountable subset of $A$. For $|K|=\aleph_{1}$ is immediate. Assume that $|K|>\aleph_{1}$. Assume that $K$ has no accumulation points in $B$. Then there exists $K^{\prime} \subset K$ such that $\left|K^{\prime}\right|=\aleph_{1}$ with no complete accumulation points in $B$, contradiction.

Now, assume that (v) holds, we want to prove (i). Let $\mathcal{U}$ be an open cover of $Z$, and assume, without loss of generality, that it consists of basic open sets. Since $B \times\{0\}$ is Lindelöf, we can cover it with countably many open sets from the cover $\mathcal{U}$. Denote such a cover $\mathcal{U}_{B \times\{0\}}$. Let $K \subseteq A$ such that $K \times\{1\}=$ $Z \backslash \bigcup \mathcal{U}_{B \times\{0\}}$ where $\bigcup \mathcal{U}_{B \times\{0\}} \supseteq B \times\{0\}$. We claim that $K \times\{1\} \cup \mathcal{U}_{B \times\{0\}}$ is a countable subcover of $\mathcal{U}$ for $Z$. Indeed, $K \times\{1\} \cap\left(\bigcup \mathcal{U}_{B \times\{0\}}\right)=\varnothing$, so $K \times\{1\}$ 
has no accumulation points in $B \times\{0\}$ and so $K$ has no accumulation points in $B$.

For (i) $\Rightarrow$ (vi), assume that $Z$ is Lindelöf. Let $U$ open set containing $B$. If $|A \backslash U|>\aleph_{0}$, let $U_{B}=(\hat{U} \cap Z) \supseteq B \times\{0\}$ be an open set in $Z$. Then $U_{B}$ with the points of $(A \times\{1\}) \backslash U_{B}$ has no countable subcover, a contradiction.

Assume that (vi) holds, we want to prove (v). Let $K$ be uncountable subset of $A$ with no accumulation points in $B$, then there exists an open set $U \supset$ $B \times\{0\}$ which misses $K \times\{1\}$. Then $|A \backslash U|>\aleph_{0}$.

The equivalence between (vi) and (vii) is immediate.

Corollary 3.10. Let $X$ be hereditarily Lindelöf and $Z \subseteq X \times_{a d} 2$. The following statements are equivalent:

(i) $Z$ is Lindelöf;

(ii) every uncountable subset $K$ of $A$, with $\mathrm{cf}|\mathrm{K}|>\aleph_{0}$, has a complete accumulation point in $B$;

(iii) every uncountable subset $K$ of $A$, with $|K|$ regular cardinal has a complete accumulation point in $B$;

(iv) every uncountable subset $K$ of $A$, with $|K|=\aleph_{1}$ has a complete accumulation point in $B$;

(v) every uncountable subset $K$ of $A$ has an accumulation point in $B$;

(vi) for every open subset $U$ in $X$ containing $B$, we have $|A \backslash U| \leq \aleph_{0}$

(vii) for every closed subset $F$ in $X$ that misses $B$, we have $|A \cap F| \leq \aleph_{0}$.

Corollary 3.11. Let $Z \subseteq X \times_{a d} 2$. If

(i) $B$ is Lindelöf and

(ii) for every closed subset $F$ of $X$ that misses $B$, we have $|A \cap F| \leq \aleph_{0}$, then $Z$ is Lindelöf.

Moreover, under the additional assumption that $A$ is uncountable, $Z$ is not metrizable.

Proof. From Theorem 3.9 it follows that $Z$ is Lindelöf, and since $A$ is uncountable, any uncountable subset of $A$ has accumulation points in $B$. Hence $A$ is not $\sigma$-discrete in $A \cup B$. By Proposition 3.5, it follows that $Z$ is not metrizable.

The following example shows that the restriction on the cofinality is needed.

Example 3.12 (Watson [15]). Let $X=[0,1]$ and assume that $2^{\aleph_{0}}>\aleph_{\omega}$. Consider subsets of $[0,1], A_{n}$ 's, such that $\left|A_{n}\right|=\aleph_{n}$ and $A_{n} \subset\left[\frac{1}{n+1}, \frac{1}{n}\right]$. Take $A=\bigcup_{n \in \omega} A_{n}, B=(0,1]$ and $Z=(A \times\{1\}) \cup(B \times\{0\}) \subset[0,1] \times_{a d} 2$. Let $Z_{n}=Z \cap\left(\left[\frac{1}{n}, 1\right] \times\{2\}\right)$. Then for every $n \in \omega, Z_{n}$ is compact, so $Z=\bigcup_{n \in \omega} Z_{n}$ is $\sigma$-compact, thus Lindelöf. However $A$ has no complete accumulation points in $B$, but every uncountable subset of $A$ has accumulation points in $B$.

Corollary 3.13 (Michael, Corson [3] (see olso [8])). $Z \subseteq[0,1] \times_{a d} 2$ is Lindelöf if and only if every uncountable subset of $A$ has an accumulation point in $B$.

The Michael line and its relatives are subspaces of $[0,1] \times a d ~ 2$ of a certain kind: 
Definition 3.14. If $Z \subseteq X \times_{a d} 2$ where $A$ and $B$ are disjoint, then we say that $Z$ is a Michael-type line. Assume $X=[0,1]$ unless stated otherwise.

Corollary 3.7 gives us a necessary and sufficient condition for the metrizability of a subspace of Alexandroff Duplicate. In the next result we give a different condition for metrizability, which takes into account only the size of the subspace $A$.

Proposition 3.15. Under $\mathrm{MA}_{\aleph_{1}}$, if $A, B \subset[0,1]$ such that $|A| \leq \aleph_{1}$, then the Michael-type line is metrizable.

Proof. $\mathrm{MA}_{\aleph_{1}}$ implies that every subset of the reals of cardinality at most $\aleph_{1}$ is a Q-set. Thus $A$ is the union of countably many subsets closed in $A \cup B$, i.e., $A=\bigcup_{n \in \omega} C_{n}$. Since $A \cap B=\varnothing$ and $C_{n}$ 's are closed in $A \cup B$, we have that for each $n \in \omega, C_{n}$ has no accumulation point in $B$. By Corollary 3.7 it follows that $Z$ is metrizable.

Example 3.16 (Michael, Corson [12]). Let $A=\mathbb{P}$ and $B=\mathbb{Q}$. Call this Michael-type line the Michael line LN ${ }^{\text {mic }}$.

Now we show some property of the Michael-type line $L^{\text {mic }}$.

Lemma 3.17. Let $Z \subseteq X \times_{a d}$. If

(i) $X$ hereditarily paracompact,

(ii) ind $B=0$,

(iii) $A$ is not $\sigma$-discrete in $A \cup B$,

then $Z$ is zero-dimensional, hereditarily paracompact but not metrizable.

Proof. Since ind $B=0$ applying Theorem 2.13, follow that $Z$ is zero-dimensional. Moreover $A$ is not a countable union of sets with no accumulation points in $B$. By Proposition 3.5, follow that $Z$ is not metrizable. From Proposition 2.8 it follows that $Z$ is hereditarily paracompact.

Corollary 3.18. LN $^{\text {mic }}$ is zero-dimensional, hereditarily paracompact but not metrizable.

Note that Corollary 1.20 can be restate as follows:

Corollary 3.19 (Wille [16]). LN ${ }^{\text {mic }}$ cannot be retracted onto $\mathbb{Q} \times\{0\}$.

Example 3.20 (Michael [12]). Choose a Bernstein partition [0,1] $=A \cup B$ (that is, neither $A$ nor $B$ contains an uncountable compact subset). Call this Michael-type line LN ${ }^{\mathrm{mcb}}$, the Michael-Bernstein line

Corollary 3.21 (see also Tanaka [13]). LNmcb is Lindelöf space but not metrizable.

Proof. Let $F$ be a closed subset of $X$ such that $F \cap B=\varnothing$. Since $F$ is a compact contained in $A$, then $|F| \leq \aleph_{0}$. Lemma 3.11 ends the proof.

Corollary 3.22 (Dow [5]). Under $\mathrm{MA}_{\aleph_{1}}, \mathrm{LN}^{\mathrm{mcb}}$ is a Lindelöf first countable space which is not metrizable but all of whose subspaces of cardinality $\aleph_{1}$ are metrizable. 
Proof. Apply Proposition 2.4, Corollary 3.15 and Corollary 3.21.

Next we provide a sufficient condition for $Z \subseteq X \times_{a d} 2$ to have a product with the irrationals that fails to be normal. In the following, unless stated otherwise, we assume that the $X$ is Tychonoff.

Proposition 3.23. Let $A, B$ and $C$ subsets of a topological space $X$. If

(i) $C$ is separable,

(ii) $A \subseteq C$ and $B \cap C=\varnothing$,

(iii) $A$ is not union of countably many subsets with no accumulation points in $B$,

then $Z$ not metrizable, and the product of the Michael-type line $Z$ with $C$ is not normal.

Proof. Let $K=\{((a, 1), a): a \in A\}$ and $L=(B \times\{0\}) \times C$ be subsets in $Z \times C$. First of all we prove that $K^{c}$ is an open set in $Z \times C$. Indeed, let $b \in B, c \in C$, and $((b, 0), c) \in K^{c}$. Since $C \cap B=\varnothing$, then $b \neq c$, and so, there exists $U$ and $V$ disjoint open sets in $X$ such that $b \in U$ and $c \in V$. Hence $(\hat{U} \cap Z) \times(V \cap C)$ is an open set in $Z \times C$ contained in $K^{c}$. Now, let $a \in A, c \in C \backslash\{a\}$, and $((a, 1), c) \in K^{c}$. Then there exists $U \in \tau_{X}$ such that $c \in U$ and $a \notin U$. Then $\{(a, 1)\} \times(U \cap C)$ is an open set in $Z \times C$ containing $((a, 1), c)$ and contained in $K^{c}$. Thus $K$ is closed in $Z \times C, K$ and $L$ are disjoint closed sets in $Z \times C$. Suppose these sets were separated by disjoint open sets $U$ and $V$ respectively. Each element $((a, 1), a)$ has a neighborhood of the form $\{(a, 1)\} \times U_{a} \subseteq U$ with $U_{a}$ open set in $C$ containing $a$. Let $D$ be the countable dense set in $C$. Take $d_{a} \in U_{a} \cap D$. Now, $A$ is not the union of countably many subsets with no accumulation points in $B$, then we can find a net $\left\{a_{\sigma}: \sigma \in \Sigma\right\} \subset A$ accumulating to $b_{0} \in B$ and $d \in D$ so that $d_{a_{\sigma}}=d$ for every $\sigma \in \Sigma$. Then $\left(\left(a_{\sigma}, 1\right), d\right)$ accumulates to $\left(\left(b_{0}, 0\right), d\right)$ with $\left(\left(a_{\sigma}, 1\right), d\right) \in U$ and $\left(\left(b_{0}, 0\right), d\right) \in V$. Thus we have found $\left(\left(b_{0}, 0\right), d\right) \in \bar{U} \cap V$ which is impossible.

Corollary 3.24. Let $A, B$ and $C$ be subsets of a topological space $X$. If

(i) $C$ is separable,

(ii) $A \subseteq C$ and $B \cap C=\varnothing$,

(iii) every uncountable subset $K$ of $A$ has an accumulation point in $B$,

(iv) $A$ is uncountable

(v) $B$ is Lindelöf,

then the Michael-type line $Z$ is Lindelöf, not metrizable, and $Z \times C$ is not normal.

Proof. It follows directly from Theorem 3.9 and Proposition 3.23.

Corollary 3.25. Let $A$ and $B$ be subsets of a topological space $X$. If $X \backslash B$ is separable and every uncountable subset $K$ of $A$ has an accumulation point in $B$, then $Z \times(X \backslash B)$ is not normal.

Moreover, if $X$ is compact, $A$ is uncountable and $X \backslash B$ Cech-complete, then the Michael-type line $Z$ is also Lindelöf and not metrizable. 
Proof. Since $X \backslash B$ is a Čech-complete in $X$, it is a $G_{\delta}$ in $X$. Thus $B$ is an $F_{\sigma}$ of a compact space, hence Lindelöf. The statement follows from Corollary 3.24.

Corollary 3.26 (based on Michael [11]). Let $A$ and $B$ be subsets of [0,1]. If $A$ is contained in a copy of the irrationals disjoint from $B$, and $A$ is not the union of countably many subsets with no accumulation points in $B$, then the product of the Michael-type line with the irrational is not normal.

Corollary 3.27 (Michael [12]). LN $^{\text {mic }} \times \mathbb{P}$ is not normal.

Corollary 3.28 (Michael [12]). $\mathrm{LN}^{\mathrm{mcb}} \times A$ is not normal.

In order to give another example of a Lindelöf space whose product with the irrationals is not normal, we first recall another class of Michael-type lines.

Example 3.29. (Michael [12]; Burke, Davis [2]; van Douwen [14]; Lawrence [10]) Under $\mathbf{b}=\omega_{1}$, let $A \subset{ }^{\omega} \omega$ be unbounded and well-ordered in type $\omega_{1}$ by $<^{*}$. Let $B=\mathbb{Q}$. Call the Michael-type line $\mathrm{LN}^{\mathrm{mbd}}$.

Lemma 3.30. Under $\mathbf{b}=\omega_{1}$, let $C={ }^{\omega} \omega \subseteq X$ with $X$ compact and $A \subseteq{ }^{\omega} \omega$ be unbounded and well-ordered in type $\omega_{1}$ by $<^{*}, B=X \backslash C$. Then every uncountable subset of $A$ has accumulation points in $X \backslash C$.

Proof. Let $f$ be the standard homeomorphism between $\mathbb{P}$ and ${ }^{\omega} \omega$. The set $A$ is a $F_{\sigma}$ in $A \cup(X \backslash C) \subset X$ if and only if $A \subset \bigcup_{n \in \omega} K_{n}$ where $K_{n}$ 's are closed in $X$ and $\left(\bigcup_{n \in \omega} K_{n}\right) \cap(X \backslash C)=\varnothing$, i.e., $K_{n}$ is a compact subset of $\mathbb{P}$ for each $n \in \omega$. Since every compact subset of $\omega^{\omega}$ is bounded in $\omega^{\omega}$, then for each $K_{n} \subset \mathbb{P}$, there exists $f_{n} \in \omega^{\omega}$ such that $f\left(K_{n}\right) \leq^{*} f_{n}$. Since $\mathbf{b}=\omega_{1}$, take $g \in \omega^{\omega}$ such that $f_{n} \leq^{*} g$ for each $n \in \omega$. Then $g$ bounds $\bigcup_{n \in \omega} f\left(K_{n}\right)=f\left(\bigcup_{n \in \omega} K_{n}\right)$ which contains $f(A)$. Then $f(A) \cong A \subset{ }^{\omega} \omega$ is bounded. Observe that any uncountable $K \subset A$ is unbounded and then suck $K$ cannot be compact, i.e., $K$ cannot be closed in $(X \backslash C) \cup K$. Thus any uncountable $K \subset A$ has accumulation points in $X \backslash C$.

Corollary 3.31. (Michael [12]; Burke, Davis [2]; van Douwen [14]; Lawrence [10]) $\mathrm{LN}^{\mathrm{mbd}}$ is a Lindelöf space whose product with the irrationals is not normal.

Proof. Follows from Lemma 3.30, Corollary 3.25.

Problem 3.32 (implicit in Michael [12]). Is there a Lindelöf space whose product with the irrationals is not normal?

\section{REFERENCES}

[1] P. S. Alexandroff and P. S. Urysohn, Mémoire sur les espaces topologiques compacts, Verh. Akad. Wetensch. Amsterdam, 14 (1929).

[2] D. K. Burke and S. W. Davis, Subsets of ${ }^{\omega} \omega$ and generalized metric spaces, Pacific J. Math. 110(2) (1984), 273-281.

[3] H. H. Corson and E. Michael, Metrizability of certain countable unions, Illinois J. Math. 8 (1964), 351-360. 
[4] J. Dieudonné, Une généralisation des espace compact, J. de Math. Pures et Appl. 23 (1944), 65-76.

[5] A. Dow, An empty class of nonmetric spaces, Proc. Amer. Math. Soc. 104(3) (1988), 999-1001.

[6] R. Engelking, General Topology (Heldermann Verlag, Berlin 1989).

[7] V. V. Fedorčuk, Bicompacta with noncoinciding dimensionalities, Soviet Math. Doklady 9(5) (1968), 1148-1150.

[8] R. F. Gittings, Finite-to-one open maps of generalized metric spaces, Pacific J. Math. $\mathbf{5 9}(1)$ (1975), 33-41.

[9] K. Kunen, Set Theory. An Introduction to Independence Proofs, (North-Holland, Amsterdam 1980).

[10] L. B. Lawrence, The influence of a small cardinal on the product of a Lindelöf space and the irrationals, Proc. Amer. Math. Soc. 110(2) (1990), 535-542.

[11] E. Michael, Paracompactness and the Lindelöf property in finite and countable Cartesian products, Compositio Math. 23(2) (1971), 199-214.

[12] E. Michael, The product of a normal space and a metric space need not be normal, Bull. Amer. Math. Soc. 69 (1963), 375-376.

[13] Y. Tanaka, Decompositions of spaces determined by compact subsets, Proc. Amer. Math. Soc. 97(3) (1986), 549-555.

[14] E. K. van Douwen, The integers and topology, In K. Kunen and J. Vaughan, (eds.), Handbook of Set-Theoretic Topology 111-169 (North-Holland, Amsterdam, 1984).

[15] S. Watson, The Construction of Topological Spaces: Planks and Resolutions, In M. Hussek and J. van Mill (eds.), Recent Progress in General Topology, 673-757 ( NorthHolland 1992).

[16] R. J. Wille, Sur les espaces faiblement rétractiles, Ned. Akad. Weten. Proc. 57 (1954), $527-532$.

RECEIVED November 2005

ACCEPted June 2006

A. CAserta (agata.caserta@unina2.it)

Dipartimento di Matematica, Seconda Università degli Studi di Napoli, Caserta 81100, Italia

\section{S. WATSON (watson@hilbert.math.yorku.ca)}

Department of Mathematics and Statistics, York University, Toronto M3J1P3, Canada 